\title{
Strengthening at nanoscaled coherent twin boundary in f.c.c. metals
}

\author{
Pei Gu${ }^{\mathrm{a} *}$, Ming Dao ${ }^{\mathrm{b}}$ and Yuntian $\mathrm{Zhu}^{\mathrm{c}}$ \\ ${ }^{a}$ Department of Engineering Mechanics, Hohai University, Nanjing, Jiangsu 210098, China; \\ ${ }^{b}$ Department of Materials Science and Engineering, Massachusetts Institute of Technology, \\ Cambridge, MA 02139, USA; ${ }^{c}$ Department of Materials Science and Engineering, North Carolina \\ State University, Raleigh, NC 27695, USA
}

(Received 10 October 2013; accepted 14 January 2014)

\begin{abstract}
This paper analyses slip transfer at the boundary of nanoscaled growth twins in face-centred cubic (f.c.c.) metals for strengthening mechanism. The required stress for slip transfer, i.e. inter-twin flow stress, is obtained in a simple expression in terms of stacking fault energy and/or twin boundary (TB) energy, constriction energy and activation volume. For nanotwinned $\mathrm{Al}, \mathrm{Cu}$ and $\mathrm{Ni}$, inter-twin flow stress versus twin thickness remarkably shows Hall-Petch relationship. The Hall-Petch slope is rationalized for various reactions of screw and non-screw dislocations at the TB. Additionally, strengthening at the boundary of nanoscaled deformation twins in f.c.c. metals is analysed by evaluating required twinning stress. At small nanograin size, the prediction of deformation twin growth stress shows inverse grain-size effect on twinning, in agreement with recent experimental finding.
\end{abstract}

Keywords: nanostructures; dislocations; strengthening mechanisms

\section{Introduction}

Growth twins with tens of nanometres as twin thickness embedded in ultrafine grains of several hundred nanometre size, such as nanotwinned $\mathrm{Cu}(\mathrm{nt}-\mathrm{Cu})$, are introduced by pulsed electrodeposition [1-3]. Such nanotwins have been shown to be effective for enhancing strength and preserving ductility compared to nanograins without twinning, because coherent twin boundary (TB) acts as both barrier to dislocation motion and source for dislocation generation. In molecular dynamics (MD) simulations, various reactions of screw and non-screw dislocations in the forms of absorption to TB and/or transmission to adjacent twin lamella were discovered [4-6]. The role of these dislocation reactions at $\mathrm{TB}$ in strengthening was investigated in mechanistic model [7-10]. In recent slip transfer model [11], inter-twin flow stress, the required shear stress for absorption to $\mathrm{TB}$ and/or transmission to adjacent twin lamella, is quantified to be strongly dependent on twin thickness (twin density). Such inter-twin flow stress is the counterpart of intra-twin flow stress [9], which governs slip when the loop extends within a twin lamella. For nt-Cu, it was shown in Ref. [11] that the obtained inter-twin flow stress for various reactions of screw and non-screw dislocations that were observed

\footnotetext{
*Corresponding author. Email: pei_gu@yahoo.com
} 
in MD simulations [4,5] is consistent with experimental measurement of flow stress [2]. But, no study of inter-twin flow stress has been done for other nanotwinned metals.

Twinning does occur in other face centered cubic (f.c.c.) metals such as $\mathrm{Al}$ and $\mathrm{Ni}$ $[12,13]$, which have much higher stacking fault energies than $\mathrm{Cu}$. Crystallographic analysis and MD simulation showed various reactions at TB for impinging screw and non-screw dislocations $[4,5,14]$. Therefore, the study of slip transfer in nanotwinned Al (nt-Al) and nanotwinned Ni (nt-Ni) is important to understand their deformation behaviour for enhancing strength and preserving ductility. But, since there are no activation volume data for nt-Al and nt-Ni, it is impossible to directly apply the size dependent slip transfer model [11] to these nanotwins. In this study, the activation volumes of nt$\mathrm{Al}$ and nt-Ni are obtained from the concept of structural length equivalence in the unified mechanistic model [9] and then used to evaluate required stresses of slip transfers observed in MD simulations for nt-Al and nt-Ni. Similar to $\mathrm{nt}-\mathrm{Cu}$, the results show that these inter-twin flow stresses of nt-Al and nt-Ni are strongly size dependent and are in a comparable range to flow stresses of their counterparts, nanograined $\mathrm{Al}$ (nc$\mathrm{Al}$ ) and $\mathrm{Ni}$ (nc-Ni). When plotting the inter-twin flow stress versus the inverse of the square root of twin thickness, we find remarkably that the inter-twin flow stress of nt$\mathrm{Al}$ and $\mathrm{nt}-\mathrm{Ni}$, as well as $\mathrm{nt}-\mathrm{Cu}$, follows Hall-Petch relationship. Grain boundary strengthening leading to Hall-Petch relationship was discussed in [15], which established the Hall-Petch slope as the stress intensity for slip transfer to activate the slip system of adjacent grain, and was later extended to rationalize nanocrystalline materials in [16]. Comparing to grain boundary, the unique structure of nanotwin, its coherent $\mathrm{TB}$ and symmetry with respect to the other side of the TB, allows us to develop analytical expressions of flow stress for these dislocation reactions observed in MD simulations in terms of nanotwin's unique properties (TB energy, stacking fault energy and twin thickness) and investigate the cross-slip of each dislocation reaction for strengthening in detail.

Nanoscaled deformation twins are another type of twinning for strength enhancement where the twins are formed during deformation in the nanograins (less than $100 \mathrm{~nm}$ ), see [17]. For this type of TB strengthening, we discuss the formation and growth of nanoscaled deformation twins by slip and slip transfer from a proposed mechanistic model. The required stresses are obtained in a simple form in terms of stacking fault energy, TB energy, as well as the structural length scale in this case, grain size. It is shown that for small grain size, stress required for deformation twin growth is larger than stress required for the slip of partial dislocations without twinning so that twinning tendency becomes small. This prediction is in agreement with recent experimental finding of inverse grain-size effect on twinning [13].

The plan of the paper is as follows. In Section 2, we present the slip transfer model for various reactions of screw and non-screw dislocations at nanoscaled TB of aluminium, copper and nickel; associated inter-twin flow stress is quantified in terms of structural deformation properties and written into a simple generic form. In Section 3, we investigate TB strengthening in two aspects: from the slip transfer model in Section 2, we analyse inter-twin flow stresses and Hall-Petch slopes for slip transfer at the $\mathrm{TB}$ in nt-Al, nt-Cu and nt-Ni; from a nanoscaled mechanistic twinning model, we evaluate deformation twin growth stress and discuss the inverse grain-size effect on twinning. 


\section{TB slip transfer model}

\subsection{Screw dislocation}

The coherent TB and slip system in a nanotwinned f.c.c. metal are schematically illustrated in Figure 1. In the pile-up cross-slip model, Figure 2, an array of screw dislocations is lined up against the TB; under external loading, a partial dislocation disassociated from the first screw dislocation is either absorbed onto the TB with orientation angle $\phi_{1}$ or transmitted onto the slip plane of the next twin lamella with orientation angle $\phi_{2}$. At the intersection with TB, the two partials which form the screw dislocation BA are constricted in order to cross-slip [18,19]. At the initiation of cross-slip, consider a small half-circular loop of partial dislocation with radius $r$ moving onto the TB or the slip plane of adjacent twin lamella from the portion of constriction. From the previous work [7.9], the energy of the cross-slipped halfcircular loop is given as $F_{1}=0.3125 G b_{1}^{2} r \ln \left(r / r_{0}\right)$, where $b_{1}$ is the magnitude of the partial dislocation's Burgers vector and $r_{0} \approx b_{1}$ is the core cut-off radius. The energy of the initial partial dislocation segment before slip transfer, which corresponds to the half-circular loop of the cross-slipped partial dislocation, is taken to be the energy per unit length of a straight partial dislocation scaled by the initial segment length $2 r$, that is $F_{2}=2 r\left\lfloor G b_{1}^{2} \ln \left(r / r_{0}\right) /(4 \pi)\right\rfloor$. Combining these two expressions, the energy change due to the lengthening of the loop in the cross-slip process is $F=F_{1}-F_{2}=0.1533 G b_{1}^{2} r \ln \left(r / r_{0}\right)$. It is noted that rigorous mathematical treatment of the loop energy change, which is in complicated form, is not sought here; this fair approximation leads to a simple and clear expression for inter-twin flow stress, which is shown later to be consistent with experimental data. The free energy change for the cross-slip of the pile-up screw dislocations is [7,20],

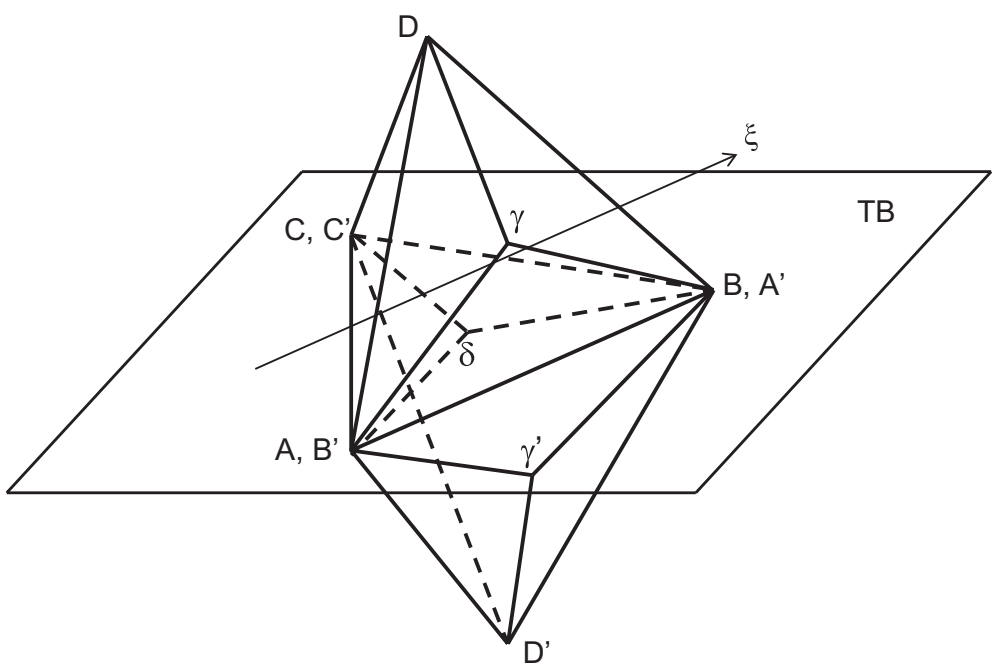

Figure 1. Dislocations in twinned f.c.c. material. In the lamella above the TB, slip system is given by Thompson tetrahedron ABCD; below the TB, slip system is given by Thompson tetrahedron $\mathbf{A}^{\prime} \mathbf{B}^{\prime} \mathbf{C}^{\prime} \mathbf{D}^{\prime}$. Dislocation is defined by clockwise Burgers circuit when sighting down the positive direction of $\boldsymbol{\xi}$, see Ref. [21]. 

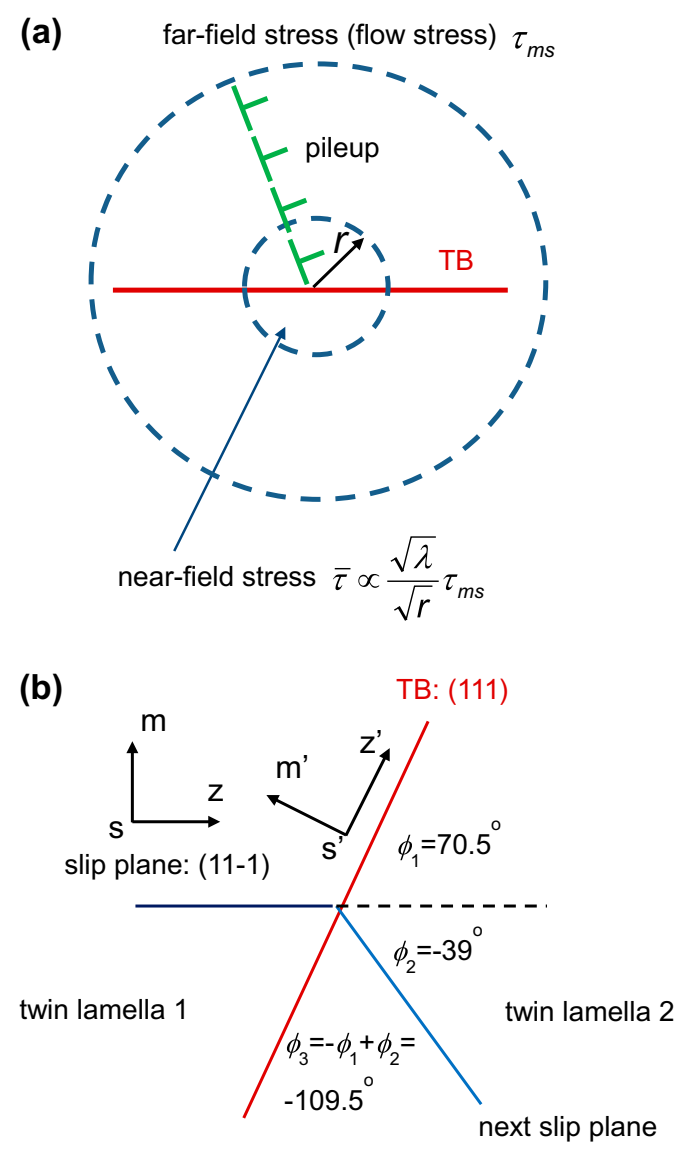

Figure 2. (colour online) (a) Pile-up dislocation slip transfer model showing the relation between the near-field stress and far-field stress. (b) Slip plane orientation: original slip plane, next slip plane and TB.

$$
\Delta F=\Phi r \ln \frac{r}{r_{0}}-H\left(r^{\frac{3}{2}}-r_{0}^{\frac{3}{2}}\right)+P\left(r^{2}-r_{0}^{2}\right)+2 U r
$$

Here, $\Phi=0.1533 G b_{1}^{2} ; P=\pi \Gamma / 2 ; \Gamma=\Gamma_{S}$ (stacking fault energy) for transmission; $\Gamma=\Gamma_{T}$ (TB energy) for absorption; $U$ is the constriction energy (energy/unit length). There should be a constant term in Equation (1) for point constriction energy [19]. It is excluded here because it does not contribute to inter-twin flow stress, which is obtained from the derivative of the free energy change as seen later. The far-field shear stress $\tau_{m s}$ to drive the screw dislocation to TB acts on the original slip plane $\mathbf{m}$ (for $\mathbf{~ m - s - z}$ coordinate system, see Figure 2(b)) and along the direction of the Burgers vector BA, which is defined by clockwise Burgers circuit when sighting down the positive dislocation line $\xi$ [21]. The shear stress near the intersection of the slip plane and the TB is the nearfield stress, given by the mode III crack tip stress field [20], 


$$
\tau_{m^{\prime} s^{\prime}}=\frac{K_{I I I}}{\sqrt{2 \pi R}} f_{I I I}(\phi)=\frac{K_{I I I}}{\sqrt{2 \pi R}} \cos \left(\frac{\phi}{2}\right),
$$

where $K_{I I I}=\sqrt{2 \pi \lambda} \tau_{m s} ; \lambda$ is twin thickness; $\phi$ is the orientation angle measured from the original slip plane, see Figure 2(b); $R$ is the distance from the intersection of slip planes along $\mathbf{z}^{\prime}$ axis (for this axis, as well as $\mathbf{m}^{\prime}$ and $\mathbf{s}^{\prime}$ axes used later, see Figure 2(b)). Note that such pile-up model resulting in stress concentration due to dislocation accumulation, which has the similarity to a crack in fracture mechanics, was also used in previous studies of cross-slip [20,22]. Consider the first cross-slipped partial dislocation's Burgers vector on the slip plane $\mathbf{m}^{\prime}$ is $\boldsymbol{b}_{\mathbf{1}}$ with its component along $\mathbf{s}^{\prime}$ axis being $b_{1 s^{\prime}}$. The work done by shear stress for the extension of $\boldsymbol{b}_{\mathbf{1}}$ is the integration of $\tau_{m^{\prime} s^{\prime}} b_{1 s^{\prime}}$ over the half-circular area of cross-slip, and using Equation (2), is given as:

$$
\int \frac{K_{I I I}}{\sqrt{2 \pi r \sin \theta}} f_{I I I}(\phi) b_{1 s^{\prime}} \mathrm{d} A=1.4 K_{I I I} f_{I I I}(\phi) b_{1 s^{\prime}}\left(r^{\frac{3}{2}}-r_{0}^{\frac{3}{2}}\right)=H\left(r^{\frac{3}{2}}-r_{0}^{\frac{3}{2}}\right) .
$$

Here, the area element for the half-circular area is $\mathrm{d} A=r \mathrm{~d} r \mathrm{~d} \theta$ and $0 \leq \theta \leq \pi$. The factor in Equation (3), $r \sin \theta$, is the distance along the $\mathbf{z}^{\prime}$ axis between the area element and the intersection of slip planes. From Equation (3), we obtain $H$ in Equation (1),

$$
H=1.4 K_{\text {III }} f_{I I I}(\phi) b_{1 s^{\prime}} .
$$

Using $K_{I I I}$ defined above and $b_{1 s^{\prime}}=b_{1} \cos 30^{\circ}$, we get $H=1.98 \sqrt{\pi \lambda} \tau_{m s}$ $b_{1} \cos (\phi / 2) \cos 30^{\circ}$.

Considering slip transfer as the non-homogeneous creation of a half-circular loop on the cross-slipped plane [9-11], the cross-slipped loop activates when the free energy change reaches maximum at activation size: $\partial \Delta F / \partial r=0$ at $r=r_{a}$. Here, $r_{a}$ is the activation size given by $r_{a}=\sqrt{2 V /(\pi b)}$, which depends on both twin thickness and grain size. The activation volume $V$ for nt-Cu was characterized in Figure 7 of Ref. [9] for $\mathrm{nt}-\mathrm{Cu}$. The above maximum condition leads to,

$$
\tau=\frac{0.06725 G b_{1}}{\sqrt{\lambda r_{a}} \cos (\phi / 2)}\left(1+\frac{1}{2} \ln \frac{r_{a}}{r_{0} e}+\frac{r_{a} P}{\Phi}+\frac{U}{\Phi}\right) .
$$

Here, $\tau \equiv \tau_{m s}$, the required shear stress for cross-slip which is referred to as inter-twin flow stress. The absorption flow stress is obtained by taking $\phi=\phi_{1}$, and transmission flow stress is obtained by taking $\phi=\phi_{2}$, in Equation (5). In transmission, a trailing partial follows the leading partial. In absorption, when first twinning partial is activated, the second twinning partial is repealed to move in the opposite direction $\phi=-\pi+\phi_{1}$ $[4,6,8]$. The first twinning partial is chosen along the direction $\phi_{1}$ because the driving force $\tau_{m^{\prime} s^{\prime}} b_{1 s^{\prime}}$ is larger in this direction than another twinning direction $\phi_{3}=-\pi+\phi_{1}$. If the two twinning partials are activated at the same time, the energy change associated with both partials needs to be accounted for. The first and third terms in the right side of Equation (1) are scaled by a multiplier 2, whereas the second term needs to include the work done by shear stresses along both twinning directions. Following the above maximizing procedure, the inter-twin flow stress in the case of both twinning partials' activation at the same time is

$$
\tau=\frac{0.1345 G b_{1}}{\sqrt{\lambda r_{a}}\left[\cos \left(\phi_{1} / 2\right)+\sin \left(\phi_{1} / 2\right)\right]}\left(1+\frac{1}{2} \ln \frac{r_{a}}{r_{0} e}+\frac{r_{a} P}{\Phi}+\frac{U}{2 \Phi}\right) .
$$


Our calculation shows shear stress from Equation (5) is close to that from Equation (6), so we take Equation (5) as the absorption flow stress. Also note that when the obtained inter-twin flow stress and activation size are substituted into Equation (1), we obtain activation energy, which was discussed in Ref. [11].

\subsection{Non-screw dislocation}

Obeying the conservation of Burgers vectors, non-screw dislocations' cross-slip to TB and/or next twin lamella is possible. The reactions for $60^{\circ}$ degree dislocation at the TB were discussed in MD simulation [5]. We calculate the inter-twin flow stress resulting from $60^{\circ}$ degree dislocation's slip transfer by extending the approach for screw dislocation discussed above. According to the dislocation definition [21], for DA, the shear stress acting along its Burgers vector direction on the original slip plane $\mathbf{m}$ drives the dislocation to the TB; when reversing the dislocation's Burgers vector to $\mathbf{A D}$, the shear stress is reversed also. To calculate the work done by shear stress in slip transfer, we project the shear stress on a cross-slipped plane $\mathbf{m}^{\prime}$ into the axes $\mathbf{s}^{\prime}$ and $\mathbf{z}^{\prime}$ shown in Figure 2(b). The shear stress along the axis $\mathbf{s}^{\prime}$ on the cross-slipped plane is the resolved shear stress from $\tau_{m s}=\tau / 2$ on the original slip plane before cross-slip, and the shear stress along the axis $\mathbf{z}^{\prime}$ on the cross-slipped plane is the resolved shear stress from $\tau_{m z}=\tau \sqrt{3} / 2$. on the original slip plane before cross-slip. For DA, in the coordinate system in Figure 2(b), the sign of $\tau$ is positive; for $\mathbf{A D}$, the sign of $\tau$ is negative. The shear stress along the axis $\mathbf{s}^{\prime}$ near the intersection of the slip plane and the TB (nearfield stress) is given by Equation (2). The shear stress along the axis $\mathbf{z}^{\prime}$ near the intersection of the slip plane and the TB (near-field stress) is obtained from mode II crack tip field $[20,23]$ as

$$
\tau_{m^{\prime} z^{\prime}}=\frac{K_{I I}}{\sqrt{2 \pi R}} f_{I I}(\phi)=\frac{K_{I I}}{\sqrt{2 \pi R}}\left[\frac{1}{4} \cos \left(\frac{\phi}{2}\right)+\frac{3}{4} \cos \left(\frac{3 \phi}{2}\right)\right],
$$

where $K_{I I}=\sqrt{2 \pi \lambda} \tau_{m z}$. Consider the first cross-slipped partial dislocation's Burgers vector on the slip plane $\mathbf{m}^{\prime}$ is $\boldsymbol{b}_{\mathbf{1}}$ with its components being $b_{1 s^{\prime}}$ and $b_{1 z^{\prime}}$ along the axes $\mathbf{s}^{\prime}$ and $\mathbf{z}^{\prime}$, respectively. The work done by shear stress for the extension of $\boldsymbol{b}_{\mathbf{1}}$ is the integration of $\tau_{m^{\prime} s^{\prime}} b_{1 s^{\prime}}+\tau_{m^{\prime} z^{\prime}} b_{1 z^{\prime}}$ over the half-circular area of cross-slip, and using Equations (2) and (7), is given as:

$$
\int\left(\frac{K_{I I I}}{\sqrt{2 \pi r \sin \theta}} f_{I I I}(\phi) b_{1 s^{\prime}}+\frac{K_{I I}}{\sqrt{2 \pi r \sin \theta}} f_{I I}(\phi) b_{1 z^{\prime}}\right) \mathrm{d} A=H\left(r^{3 / 2}-r_{0}^{3 / 2}\right),
$$

where $H=1.4\left[K_{I I I} f_{I I I}(\phi) b_{1 s^{\prime}}+K_{I I} f_{I I}(\phi) b_{1 z^{\prime}}\right]$. The area element $\mathrm{d} A$ in Equation (8) was given in Section 2.1. For cross-slip to more than one cross-slip direction in the dislocation reaction, the $H$ is the sum of the contribution of each cross-slip direction,

$$
H=\sum_{j} 1.4\left[K_{I I I} f_{I I I}\left(\phi_{j}\right) b_{1 s^{\prime} j}+K_{I I} f_{I I}\left(\phi_{j}\right) b_{1 z^{\prime} j}\right]
$$

where the cross-slip direction $\phi_{j}=\phi_{1}, \phi_{2}, \phi_{3}$, as shown in Figure 2(b). When the dislocation reaction leads to partials' cross-slip to all three directions, the free energy change is written in the form similar to Equation (1), 


$$
\Delta F=3 \Phi r \ln \frac{r}{r_{0}}-H\left(r^{\frac{3}{2}}-r_{0}^{\frac{3}{2}}\right)+P^{*}\left(r^{2}-r_{0}^{2}\right)+2 U r,
$$

where $P^{*}=\pi\left(2 \Gamma_{T}+\Gamma_{S}\right) / 2$. If the cross-slip directions are 2 , say $\phi_{2}$ and $\phi_{3}$, the sum in Equation (9) is for the contributions of these two directions; the multiplier in the first term on the right side of Equation (10) is 2; and $P^{*}=\pi\left(\Gamma_{T}+\Gamma_{S}\right) / 2$. If the cross-slip direction is 1, the sum in Equation (9) is for the contribution of this direction; the multiplier in the first term on the right side of Equation (10) is $1 ; P^{*}=\pi \Gamma_{S} / 2\left(\phi_{2}\right)$ or $P^{*}=\pi \Gamma_{T} / 2\left(\phi_{1}\right.$ or $\left.\phi_{3}\right)$. Similar to the screw dislocation case, the inter-twin flow stress is obtained by maximizing Equation (10) at the activation size.

Reactions observed in MD simulations [5] for non-screw dislocations in $\mathrm{Al}, \mathrm{Cu}$ and $\mathrm{Ni}$ are used to evaluate associated inter-twin flow stresses. For non-screw dislocation $\mathbf{D A}$ in nt-Al and nt-Cu, the reaction at TB results in a twinning partial $\mathbf{C} \boldsymbol{\delta}$ and two partials on the slip plane of adjacent twin lamella $\gamma^{\prime} \mathbf{D}^{\prime}$ (leading) $+\mathbf{A}^{\prime} \gamma^{\prime}$ (trailing). We consider that the required shear stress for cross-slip activates the twinning partial $\mathbf{C} \boldsymbol{\delta}$ and the leading partial $\gamma^{\prime} \mathbf{D}^{\prime}$. Since the two are $90^{\circ}$ twinning partials, using $K_{I I}$ and $f_{I I}$ in Equation (7) and the cross-slipped Burgers vectors' components along $\mathbf{z}^{\prime}$ axes of the two cross-slipped planes, we obtain from Equation (9),

$$
H=1.4 K_{I I} f_{I I}\left(\phi_{2}\right) b_{1}-1.4 K_{I I} f_{I I}\left(\phi_{3}\right) b_{1}=3.665 \sqrt{\lambda} \tau b_{1} .
$$

For $\mathbf{D A}$ in nt-Ni, the reaction at TB results in a twinning partial $\boldsymbol{\delta} \mathbf{A}$ and a partial $\boldsymbol{\gamma}^{\prime} \mathbf{D}^{\prime}$ on the slip plane of adjacent twin lamella, plus sessile dislocations at the intersection of the slip planes and TB 1/6[ $\left.\begin{array}{lll}0 & 0 & 1\end{array}\right]+1 / 18\left[\begin{array}{lll}1 & 1 & 1\end{array}\right]$. The sessile dislocations do not contribute to the free energy change in Equation (10). Using $K_{I I}, K_{I I I}, f_{I I}$ and $f_{I I I}$ in Equations (2) and (7) with the cross-slipped Burgers vectors' components along $\mathbf{s}^{\prime}$ and $\mathbf{z}^{\prime}$ axes, we obtain from Equation (9),

$$
H=1.4 K_{I I} f_{I I}\left(\phi_{2}\right) b_{1}-1.4 K_{I I} f_{I I}\left(\phi_{3}\right) \frac{1}{2} b_{1}+1.4 K_{I I I} f_{I I I}\left(\phi_{3}\right) \frac{\sqrt{3}}{2} b_{1}=3.665 \sqrt{\lambda} \tau b_{1} .
$$

Since both reactions have the same initial cross-slip loop number for activation, 2, and the same $H$, we obtain the inter-twin flow stress for them from maximizing the free energy in Equation (10),

$$
\tau=\frac{0.1115 G b_{1}}{\sqrt{\lambda r_{a}}}\left(1+\frac{1}{2} \ln \frac{r_{a}}{r_{0} e}+\frac{r_{a} P^{*}}{2 \Phi}+\frac{U}{2 \Phi}\right),
$$

where $P^{*}=\pi\left(\Gamma_{T}+\Gamma_{S}\right) / 2$.

For non-screw dislocation AD in nt-Al, the reaction at TB results in two twinning partials, $\boldsymbol{\delta} \mathbf{C}$ which glides along $\phi_{3}$ and $\mathbf{A} \boldsymbol{\delta}$ which glides along $\phi_{1}$, plus a sessile dislocation $1 / 2\left[\begin{array}{lll}\overline{1} & \overline{1} & 0\end{array}\right]$. For $\mathbf{A D}$ in nt-Cu and nt-Ni, the reaction at TB results in a partial $\boldsymbol{\gamma}^{\prime} \mathbf{A}^{\prime}$ on the slip plane of adjacent twin lamella, plus sessile dislocations $1 / 3\left[\begin{array}{lll}0 & 0 & 1\end{array}\right]+1 / 9\left[\begin{array}{lll}1 & 1 & 1\end{array}\right]$. The inter-twin flow stress for AD can be obtained from Equation (10) in a similar way as that for DA. For AD in nt-Al,

$$
\tau=\frac{0.1164 G b_{1}}{\sqrt{\lambda r_{a}}}\left(1+\frac{1}{2} \ln \frac{r_{a}}{r_{0} e}+\frac{r_{a} P^{*}}{\Phi}+\frac{U}{\Phi}\right),
$$

where $P^{*}=\pi \Gamma_{T} / 2$. Following the argument in Section 2.1 for the case that both crossslip directions $\phi_{1}$ and $\phi_{3}$ are involved, we consider three cases: (i) $\boldsymbol{\delta} \mathbf{C}$ is activated first 
along $\phi_{3}$; (ii) A $\mathbf{\delta}$ is activated first along $\phi_{1}$; (iii) both partials glide at the same time. Equation (14) is for the case of smallest inter-twin flow stress among the three, the case (i). For $\mathbf{A D}$ in $\mathrm{nt}-\mathrm{Cu}$ and $\mathrm{nt}-\mathrm{Ni}$,

$$
\tau=\frac{0.0855 G b_{1}}{\sqrt{\lambda r_{a}}}\left(1+\frac{1}{2} \ln \frac{r_{a}}{r_{0} e}+\frac{r_{a} P^{*}}{\Phi}+\frac{U}{\Phi}\right),
$$

where $P^{*}=\pi \Gamma_{S} / 2$.

Combining above solutions as well as those in Sections 2.1, the generic expression for inter-twin flow stress is written as:

$$
\tau=\frac{A G b_{1}}{\sqrt{r_{a}}}\left(1+\frac{1}{2} \ln \frac{r_{a}}{r_{0} e}+\frac{r_{a} P}{k \Phi}+\frac{U}{k \Phi}\right) \frac{1}{\sqrt{\lambda}} .
$$

Here, $k$ is the number of initial cross-slip directions in Figure 2(b). Inside the parentheses, the first two terms represent the contribution of loop growth during cross-slip, whereas the last two terms represent the contributions of TB energy, stacking fault energy and constriction energy. When using the approximation $r_{a} \approx b_{1} e$ [7.8.20], the second term is removed. It is noted that for the cases involving sessile dislocations, for subsequent incoming dislocations to cross-slip at the TB, further reactions are needed to dissolve dislocation locks; otherwise, strain hardening is expected.

\section{Evaluating strengthening at TB}

Since there are no experimental data available for the activation volume of nt-Al and nt-Ni currently, and it is also impossible to determine the parameter in the non-homogeneous nucleation model [9] for activation volume without the experimental data, it is required to estimate the activation volume for $\mathrm{nt}-\mathrm{Al}$ and $\mathrm{nt}-\mathrm{Ni}$ in order to use Equation (16). We recall the competition of grain size and twin thickness in [9]. For nanotwinned materials with large grain size, it was found that the effect of twin thickness on properties is equivalent to the effect of the grain size in nanograined materials (without twins) on properties, also see [2,24,25]. Specifically, the flow stress, activation volume and strain-rate sensitivity of nanotwins versus twin thickness provide good correlation for size dependence just like the grain size is used for these three physical parameters of nanograins without twinning. In such case, the activation volume of nanotwins is considered to be equivalent to that of nanograins, when the value of nanotwin thickness is the same as the value of nanograin size. For nc-Al and $\mathrm{nc}-\mathrm{Ni}$, there are limited data for their grain-size-dependent activation volume. The parameter in the non-homogeneous nucleation model, from which the activation volume is obtained in the entire nanometre range, was determined in Ref. [10] for nc-Al and nc-Ni. These grain-size-dependent activation volumes of nc-Al and nc-Ni obtained from the non-homogeneous nucleation model are used as twin-thickness-dependent activation volumes of $\mathrm{nt}-\mathrm{Al}$ and $\mathrm{nt}-\mathrm{Ni}$ here according to above size equivalence argument. It is noted that this treatment from the size equivalence conception is an approximation. As seen by comparing Figure 5 with Figure 7 in Ref. [9], the value of activation volume of $\mathrm{nt}-\mathrm{Cu}$ is not the same as that of $\mathrm{nc}-\mathrm{Cu}$ for the same structural length, but they are close. However, the results below show that the Hall-Petch slope is insensitive to the activation volume. 
The construction energy $U$ for $\mathrm{Cu}$ obtained in MD simulation was shown to be in the same range as that obtained from dislocation theory for recombining two partials from their equilibrium distance [26]. Here, we use the recombination energies from dislocation theory as the values of $U$ for $\mathrm{Al}, \mathrm{Cu}$ and $\mathrm{Ni}$ in numerics. Additional material properties in our calculations are as follows. Stacking fault energies for $\mathrm{Al}, \mathrm{Cu}$ and $\mathrm{Ni}$ are $0.146 \mathrm{~J} / \mathrm{m}^{2}$, $0.054 \mathrm{~J} / \mathrm{m}^{2}$ and $0.194 \mathrm{~J} / \mathrm{m}^{2}$ [27,28], respectively. The TB energy is $\Gamma_{T}=\Gamma_{S} / 2[29,30]$. Shear moduli for $\mathrm{Al}, \mathrm{Cu}$ and $\mathrm{Ni}$ are 35, 50 and $76 \mathrm{GPa}$, respectively; Burgers vectors for $\mathrm{Al}, \mathrm{Cu}$ and $\mathrm{Ni}$ are $0.25,0.255$ and $0.248 \mathrm{~nm}$, respectively.

Figures 3 and 4 show the prediction of inter-twin flow stress for nt-Al and nt-Ni against the inverse of square root of twin thickness. Also plotted in the two figures are the flow stress data for $\mathrm{nc}-\mathrm{Al}$ and nc-Ni [10,31]. The inter-twin flow stress of nt-Cu discussed in [11] is plotted in Figure 5 together with experimental data for nt- $\mathrm{Cu}$ [2] against the inverse of square root of twin thickness. The horizontal axis for the structural length in these figures is in the nanorange below $100 \mathrm{~nm}$. The flow stress of a nanotwinned material includes two portions, i.e. inter-twin flow stress and intra-twin flow stress. Intra-twin dislocation and inter-twin dislocation are two types of dislocation activities in nanotwins, and experiments suggest both exist [25]. Intra-twin flow stress drives dislocation extension inside twin lamella, whereas inter-twin flow stress drives dislocation to cross-slip the TB. For nt-Cu, comparison of the inter-twin flow stress obtained here and the intra-twin flow stress discussed in Ref. [9] with experimentally measured flow stress suggests the three are in the same range; for $\mathrm{nt}-\mathrm{Al}$ and $\mathrm{nt}-\mathrm{Ni}$, this is also expected to be true. In Figures 3 and 4, the fact that the flow stress data for nanograins are in the same range as the inter-twin flow stresses of nanotwins again

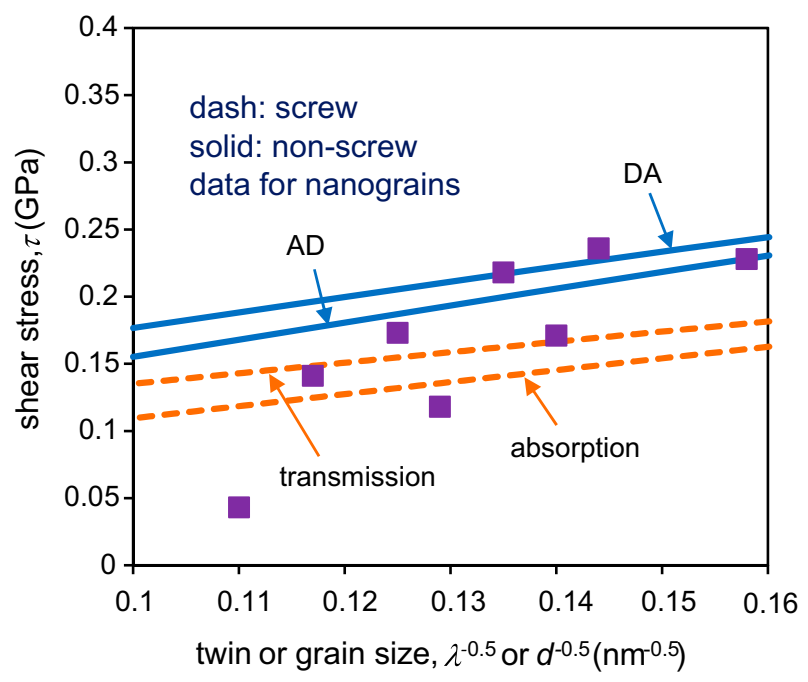

Figure 3. (colour online) Inter-twin flow stresses of screw dislocation BA and non-screw dislocations DA and AD versus twin thickness for nt-Al. The experimental data are flow stress versus grain size for nc-Al from Ref. [10]. As structural length scale, the twin thickness of nt-Al is equivalent to the grain size of nc-Al. The predicted inter-twin flow stress of nt-Al is in the same range as the flow stress data of nc-Al. The nearly straight curves suggest Hall-Petch slopes for strengthening of slip transfer, which is also true for nt-Ni and nt-Cu as shown in Figures 4 and 5. 


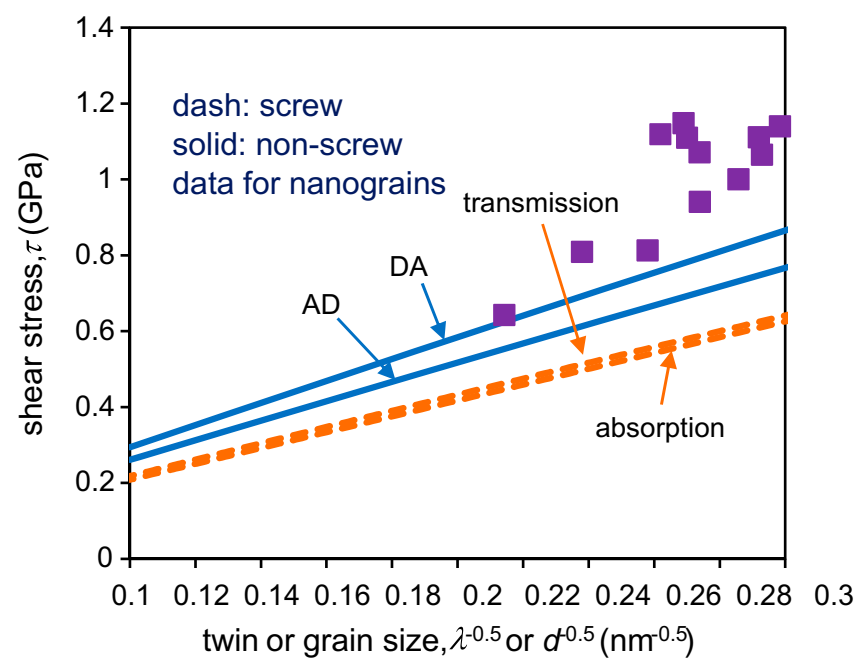

Figure 4. (colour online) Inter-twin flow stresses of screw dislocation BA and non-screw dislocations DA and AD versus twin thickness for nt-Ni. The experimental data are flow stress versus grain size for nc-Ni from Ref. [31]. The twin thickness of nt-Ni is equivalent to the grain size of nc-Ni; the predicted inter-twin flow stress of nt-Ni is in the same range as the flow stress data of nc-Ni.

points to the equivalence of grain size for a nanograined material to the twin thickness for its nanotwinned counterpart whose grain size is in the ultrafine range. On the other hand, the flow stress of a nanograined material is not exactly the same as the flow stress of its nanotwinned counterpart, because crystallographic details of the two structures are different and experimental flow stress data can be scattered. In Figure 5, the agreement of predicted curves with experimental data is better than Figures 3 and 4, since both are for nt-Cu, whereas the predicted curves are for nanotwins and the experimental data are for nanograins in Figures 3 and 4.

From the nearly straight lines for the inter-twin flow stress in Figures 3, 4 and 5, we see remarkable evidence of Hall-Petch slope for each of the dislocation reaction at the TB. In other words, the pre-expression of $(1 / \sqrt{\lambda})$ in Equation (16) is nearly a constant, weakly varying with the twin-thickness-dependent activation size $r_{a}(\lambda)$. For example, in screw dislocation transmission of nt-Al, the Hall-Petch slope is 1.13 and 1.25 for the twin thickness 39 and $70 \mathrm{~nm}$, respectively. This weak dependence proves the early treatment of using $r_{a}=b_{1} e$ when maximizing the free energy of cross-slip $[7,8,20]$. The calculated Hall-Petch slopes for dislocation reactions are listed in Table 1. Also given in the table are the averages for the reactions of nt-Al, nt-Cu and nt-Ni. For the same material, the slope for non-screw dislocation is larger than that for screw dislocation, because the edge component is more difficult to cross-slip from its crystallographic orientation. The ranking of the slope from small to large is as follows: nt-Al, $\mathrm{nt}-\mathrm{Cu}$ and nt-Ni. This is because that Equation (16) is proportional to shear modulus and the ranking of shear modulus of the three materials is the same as above ranking for the slope. Such dependence of the slope on shear modulus is consistent with previous theoretical work of micrograins and nanograins [16,32,33]. Although more dislocation reactions at the $\mathrm{TB}$ for each material in the table may be discovered later, the 


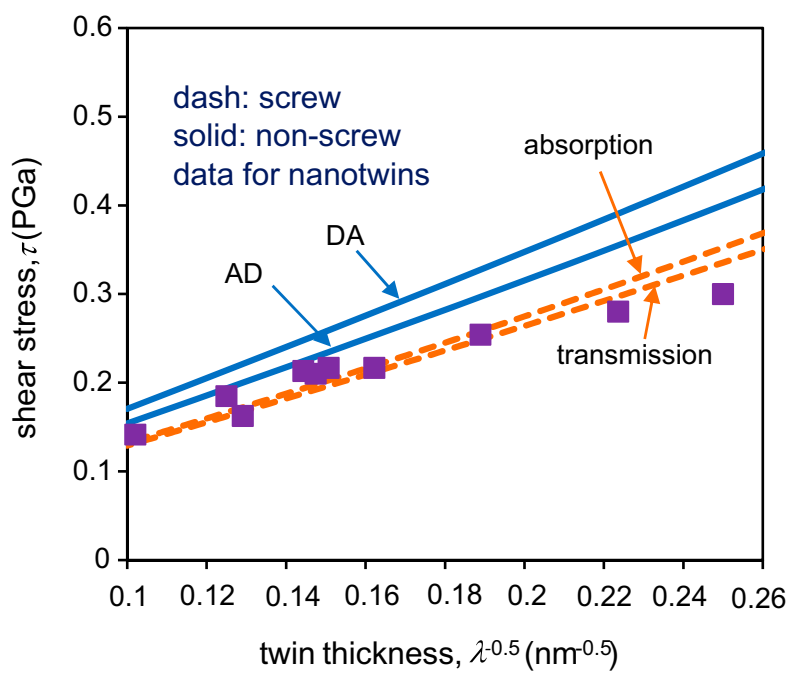

Figure 5. (colour online) Inter-twin flow stresses of screw dislocation BA and non-screw dislocations $\mathbf{D A}$ and $\mathbf{A D}$ versus twin thickness for $\mathrm{nt}-\mathrm{Cu}$. The experimental data for $\mathrm{nt}-\mathrm{Cu}$ are from Ref. [2].

Table 1. Hall-Petch slope for slip transfer at nanoscaled TB $(\mathrm{GPa} \sqrt{\mathrm{nm}})$.

\begin{tabular}{lccccc}
\hline & \multicolumn{2}{c}{ Dislocations } & Average \\
\cline { 2 - 5 } & Screw transmission & Screw absorption & Non-screw AD & Non-screw DA & \\
\hline $\mathrm{Al}$ & 1.24 & 1.05 & 1.50 & 1.64 & 1.35 \\
$\mathrm{Cu}$ & 1.36 & 1.44 & 1.60 & 1.76 & 1.54 \\
$\mathrm{Ni}$ & 2.18 & 2.08 & 2.59 & 2.90 & 2.43 \\
\hline
\end{tabular}

averaged value given here is expected as a reasonable evaluation for the slope of HallPetch relationship of the nanotwinned material.

When absorption occurs and when absorbed partials extend to larger loop on the $\mathrm{TB}$, the inter-twin flow stress for the activation of the cross-slip no longer drives the loop extension. Instead, the extension on the TB is the competition of the TB energy, loop's self-energy and the interaction of different portions of the larger loop, comparable to the grain size. Similar to the approach for the intra-granular flow stress [34], the required shear stress for the large extension on the TB can be evaluated. When a partial extends on the TB across the entire grain from one side to the opposite side, or two cross-slipped partials in the middle of the TB extend in opposite directions across it, the required shear stress is (taking $\beta=1$ in Ref. [34])

$$
\tau_{m}=\frac{\Gamma_{T}}{b}+0.306 \frac{G b}{d} .
$$

Here, $d$ is grain size. The process causes TB migration: one side of the twin lamella decreases by one atomic layer and another side increases by one atomic layer. In the case of deformation twins $[17,35,36]$, above $\tau_{m}$ is deformation twin growth stress or 
deformation twin reduction stress in nanograins. Asaro and Suresh in [7] proposed a deformation twin initiation criteria based on the generalized stacking fault energy curve and showed that deformation twins of $\mathrm{Al}, \mathrm{Cu}$ and $\mathrm{Ni}$ are achievable in comparison with partials' glide without twinning. Among various pathways, the formation of a nanoscaled deformation twin can be done by successful grain boundary emission of partials extending in parallel adjacent slip planes to the opposite grain boundary consecutively; or a two-step cross-slip of a partial at the TB: $\mathbf{B} \boldsymbol{\gamma}=\mathbf{B} \boldsymbol{\delta}+\boldsymbol{\delta} \boldsymbol{\gamma} ; \boldsymbol{\delta} \boldsymbol{\gamma}=\boldsymbol{\delta} \mathbf{B}+\mathbf{B} \boldsymbol{\gamma}$, where $\mathbf{B} \boldsymbol{\delta}$ and $\boldsymbol{\delta} \mathbf{B}$ extend in the opposite directions across the grain under shear stress to grow the twin by one atomic layer and $\mathbf{B} \boldsymbol{\gamma}$ in the second reaction extends one atomic layer further in the original slip plane of the matrix (untwined region) to continue this twinning process [37]. Here, we use above model for nanoscaled deformation twins to examine their required stresses. Obviously, in this twinning mechanism, the structural length for nanoscaled deformation twin formation and growth is grain size, as seen in Equation (17). However, Equation (17) is not the required stress for deformation twin formation, which is the stress to create the first layer, the stacking fault, across the entire grain and which is given from the approach in Ref. [34] as

$$
\tau_{t}=\frac{\Gamma_{S}}{b}+0.306 \frac{G b}{d} \text {. }
$$

Previous work [12,38] gave similar functional form as Equation (18), but did not determine the slope for gain size dependence in twin formation. Using material properties given above, for $\mathrm{Al}, \tau_{t}=1036 \mathrm{MPa}$ when $d=6 \mathrm{~nm}$; for $\mathrm{Cu}, \tau_{t}=298 \mathrm{MPa}$ when $d=46 \mathrm{~nm}$; for $\mathrm{Ni}, \tau_{t}=1039 \mathrm{MPa}$ when $d=23 \mathrm{~nm}$. These calculated values for $\tau_{t}$ from Equation (18) are consistent with those reported in Ref. [17]. The nanograins' flow stress for partials' glide without twinning is [31].

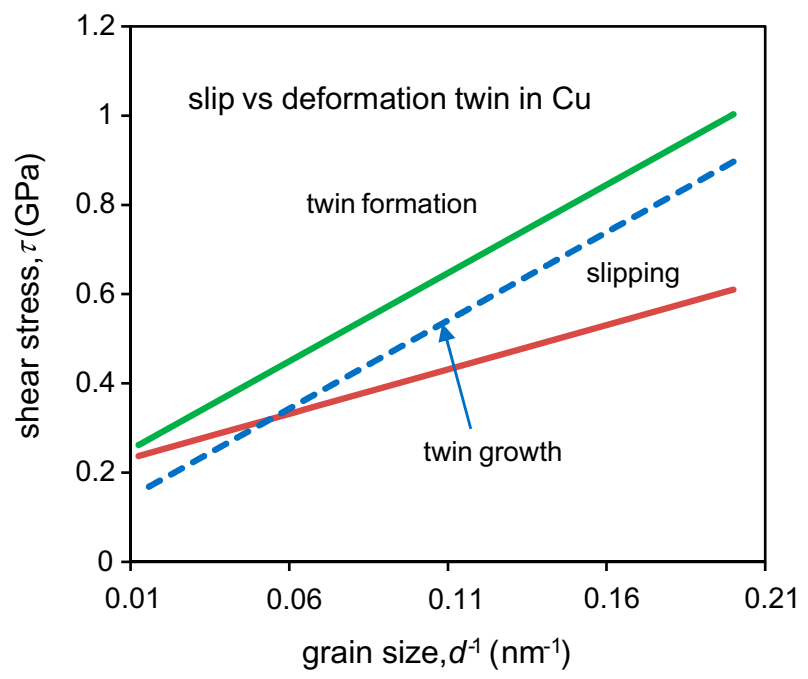

Figure 6. (colour online) Required stresses for deformation twin formation and growth versus required stress for the slip of partial dislocation without twinning in nanograined $\mathrm{Cu}$. For small grain size, stress required for deformation twin growth is larger than stress required for the slip of partial dislocation such that twinning tendency becomes small. 


$$
\tau_{f}=\frac{\Gamma_{S}}{b}+0.156 \frac{G b}{d} .
$$

The three flow stresses above, $\tau_{m}, \tau_{t}$ and $\tau_{f}$, follow the generalized Hall-Petch relationship [39], $\tau=k_{1}+k_{2} / d^{\alpha}$. In agreement with previous observation, the slope of the deformation twin formation stress is larger than that of the flow stress for partials' glide without twinning in nanograins [17], but is smaller than the slope of Hall-Petch relationship for deformation twins in micrograins [35]. The three required stresses for $\mathrm{Cu}$ are plotted versus grain size in Figure 6, which shows that for small grain size, stress required for deformation twin growth is larger than stress for the slip of partials without twinning. Hence, twinning tendency becomes small for small grain size, in agreement with experimental finding of inverse grain-size effect on twinning [13]. Such inverse grain-size effect can be explained by examining Equations (17) and (19), in which, for small grain size, the grain-size-dependent terms are dominant and the slope for twin growth is larger than the slope for the slip of partials without twinning. The transition grain size is estimated from the two equations as $d \propto G b^{2} /\left(\Gamma_{S}-\Gamma_{T}\right)$.

\section{Concluding remark}

In summary, the present mechanistic model elaborates the role of slip and slip transfer at nanoscaled growth and deformation twin boundaries in strengthening. It provides a means to evaluate inter-twin flow stress and activation energy for dislocation reactions at the TB. Quantified inter-twin flow stress of nanoscaled growth twins, as well as previously studied intra-twin flow stress [9], is related to activation volume, strain-rate sensitivity, nanostructural size, as well as other dislocation properties such that the strengthening mechanisms of nanotwins can be investigated through coupled modelling and experiment for optimizing performance. The influence of slip as well as slip transfer to the formation and growth of nanoscaled deformation twins is analysed, and the required stresses for their formation and growth are examined within the mechanistic model to show the inverse grain-size effect on twinning.

\section{References}

[1] K. Lu, L. Lu and S. Suresh, Science 324 (2009) p.349.

[2] L. Lu, X. Chen, X. Huang and K. Lu, Science 323 (2009) p.607.

[3] L. Lu, R. Schwaiger, Z.W. Shan, M. Dao, K. Lu and S. Suresh, Acta Mater. 53 (2005) p.2169.

[4] Z.H. Jin, P. Gumbsch, E. Ma, K. Albe, K. Lu, H. Hahn and H. Gleiter, Scr. Mater. 54 (2006) p.1163.

[5] Z.H. Jin, P. Gumbsch, K. Albe, E. Ma, K. Lu, H. Gleiter and H. Hahn, Acta Mater. 56 (2008) p.1126.

[6] T. Zhu, J. Li, A. Samanta, H.G. Kim and S. Suresh, PNAS 104 (2007) p.3031.

[7] R.J. Asaro and S. Suresh, Acta. Mater. 53 (2005) p.3369.

[8] R.J. Asaro and Y. Kulkarni, Scr. Mater. 58 (2008) p.389.

[9] P. Gu, M. Dao, R.J. Asaro and S. Suresh, Acta Mater. 59 (2011) p.6861.

[10] P. Gu and M. Dao, Appl. Phys. Lett. 102 (2013) p.091904.

[11] P. Gu, M. Dao and S. Suresh, Acta Mater. (2014) Available at http://dx.doi.org/10.1016/ j.actamat.2013.12.028 
[12] M.W. Chen, E. Ma, K.J. Hemker, H.W. Sheng, Y.M. Wang and X.M. Cheng, Science 300 (2003) p.1275.

[13] X.L. Wu and Y.T. Zhu, Phys. Rev. Lett. 101 (2008) p.025503.

[14] Y.T. Zhu, X.L. Wu, X.Z. Liao, J. Narayan, L.J. Kecskés and S.N. Mathaudhu, Acta Mater. 59 (2011) p.812.

[15] R.W. Armstrong, I. Codd, R.M. Douthwaite and N.J. Petch, Philos. Mag. 7 (1962) p.45.

[16] R.W. Armstrong and P. Rodriguez, Philos. Mag. 86 (2006) p.5787.

[17] Y.T. Zhu, X.Z. Liao, X.L. Wu and J. Narayan, J. Mater. Sci. 48 (2013) p.4467.

[18] B. Escaig, Cross-slipping process in the f.c.c. structure, in Dislocation Dynamics, R.A. Rosenfeld et al, eds., McGrew-Hall, New York, 1968, p. 655.

[19] W. Püschl, Prog. Mater. Sci. 47 (2002) p.415.

[20] R.J. Asaro and J.R. Rice, J. Mech. Phys. Solids 25 (1977) p.309.

[21] J. Weertman and J.R. Weertman, Elementary Dislocation Theory, Oxford University Press, New York, 1992.

[22] R.W. Armstrong, Acta Metall. 16 (1968) p.347.

[23] T.L. Anderson, Fracture Mechanics: Fundamentals and Applications, CRC Press, Boca Raton, FL, 1995.

[24] L. Lu, M. Dao, T. Zhu and J. Li, Scr. Mater. 60 (2009) p.1062.

[25] L. Lu, T. Zhu, Y.F. Shen, M. Dao, K. Lu and S. Suresh, Acta Mater. 57 (2009) p.5165.

[26] T. Rasmussen, K.W. Jacobsen, T. Leffers, O.B. Pedersen, S.G. Srinivasan and H. Jónsson, Phys. Rev. Lett. 79 (1997) p.3676.

[27] H. Van Swygenhoven, P.M. Derlet and A.G. Frøseth, Nature Materials 3 (2004) p.399.

[28] J.P. Hirth and J. Lothe, Theory of Dislocations, Wiley, New York, 1982.

[29] Y.T. Zhu, X.Z. Liao, S.G. Srinivasan, Y.H. Zhao, M.I. Baskes, F. Zhou and E.J. Lavernia, Appl. Phys. Lett. 85 (2004) p.5049.

[30] S. Kibey, J.B. Liu, D.D. Johnson and H. Schitoglu, Appl. Phys. Lett. 91 (2007).

[31] P. Gu, B. Kad and M. Dao, Philos. Mag. Lett. 92 (2012) p.111.

[32] J.C.M. Li and Y.T. Chou, Metall. Trans. 1 (1970) p.1145.

[33] H. Conrad, Nanotechnology 18 (2007) p.325701.

[34] P. Gu, B. Kad and M. Dao, Scr. Mater. 62 (2010) p.361.

[35] M.A. Meyers, O. Vöhringer and V.A. Lubarda, Acta Mater. 49 (2001) p.4025.

[36] I.J. Beyerlein, N.A. Mara, D. Bhattacharyya, D.J. Alexander and C.T. Necker, Int. J. Plast. 27 (2011) p.121.

[37] Y.T. Zhu, J. Narayan, J.P. Hirth, S. Mahajan, X.L. Wu and X.Z. Liao, Acta Mater. 57 (2009) p.3763.

[38] K.P.D. Lagerlöf, J. Castaing, P. Pirouz and A.H. Heuer, Philos. Mag. A 82 (2002) p.2841.

[39] Q. Yu, Z.W. Shan, J. Li, X.X. Huang, L. Xiao, J. Sun and E. Ma, Nature 463 (2010) p.335. 